\title{
Growth of Superconducting Hg-1212 Very-Thin Films
}

\author{
L. Fang, S. L. Yan, T. Aytug, A. A. Gapud, B. W. Kang, Y. Y. Xie, and J. Z. Wu \\ Department of Physics and Astronomy, University of Kansas, Lawrence, KS 66045, USA
}

\begin{abstract}
High quality epitaxial $\mathrm{HgBa}_{2} \mathrm{CaCu}_{2} \mathrm{O}_{6}+\delta(\mathrm{Hg}-\mathrm{High}$ 1212) films with thickness less than $100 \mathrm{~nm}$ have been successfully synthesized using cation-exchange process. The films show the superconducting transition up to $\sim 118 \mathrm{~K}$ which is close to the intrinsic value of $124 \mathrm{~K}$ for $\mathrm{Hg}-1212$ phase, and critical current densities up to $1.1 \times 10^{7} \mathrm{~A} / \mathrm{cm}^{2}$ at $5 \mathrm{~K}$, $1.14 \times 10^{6} \mathrm{~A} / \mathrm{cm}^{2}$ at $77 \mathrm{~K}$ and $2.59 \times 10^{5} \mathrm{~A} / \mathrm{cm}^{2}$ at $100 \mathrm{~K}$ in zero field.
\end{abstract}

\section{INTRODUCTION}

The discovery of superconductivity in $\mathrm{Hg}$-based high- $\mathrm{T}_{\mathrm{c}}$ superconductors (Hg-HTSs: $\mathrm{HgBa}_{2} \mathrm{Ca}_{n-1} \mathrm{Cu}_{\mathrm{n}} \mathrm{O}_{2 \mathrm{n}+2+d}, \mathrm{n}=1-5$ ) has attracted considerable interest due to the high critical temperature $\left(T_{c}\right)$ of these materials [1,2]. Despite the difficulties associated with the high volatile nature of the $\mathrm{Hg}$ based compounds, considerable progress has been made recently in fabrication of high-quality $\mathrm{Hg}-1212$ and $\mathrm{Hg}-1223$ films [3-7]. Typically, the thickness of these films is in the range of $0.5-1 \mu \mathrm{m}$ and the minimum thickness is still above $200 \mathrm{~nm}$. For many microelectronic applications, such as optical devices, high-quality very-thin films with their thickness less than $100 \mathrm{~nm}$ are necessary. The major difficulty in fabrication of thinner films of the $\mathrm{Hg}$-based HTSs rises from the liquefying of the material during the film crystallization in the post $\mathrm{Hg}$-vapor annealing, resulting in unconnected islands when the film thickness is below certain threshold. In addition, the severe air-sensitivity of the cuprate precursor $\left(\mathrm{Ba}_{2} \mathrm{Ca}_{n-1} \mathrm{Cu}_{n} \mathrm{O}_{\mathrm{x}}\right)$ used in the currently adopted two-step process further complicates the processing and results in poor sample reproducibility even for thick films. In order to solve these problems, we have recently developed a new precursor film scheme. Instead of using un-reacted $\mathrm{Ba}_{2} \mathrm{Ca}_{n-1} \mathrm{Cu}_{\mathrm{n}} \mathrm{O}_{\mathrm{x}}$ precursor, a pre-reacted $\mathrm{Tl}_{\mathrm{y}} \mathrm{Ba}_{2} \mathrm{Ca}_{\mathrm{n}-1} \mathrm{Cu}_{\mathrm{n}} \mathrm{O}_{\mathrm{x}}$ film $(\mathrm{y}=1$ or $2, \mathrm{n}=2$ or 3 ) is subjected to the same $\mathrm{Hg}$-vapor annealing [8] to replace $\mathrm{Tl}$ by $\mathrm{Hg}$. In this cation-exchange process, an epitaxial template can be formed in the precursor film. This allows growth of very-thin $\mathrm{Hg}$-HTS films through a completely different mechanism and eliminates the airdetrimental effect since the $\mathrm{Tl}_{\mathrm{y}} \mathrm{Ba}_{2} \mathrm{Ca}_{\mathrm{n}-1} \mathrm{Cu}_{\mathrm{n}} \mathrm{O}_{\mathrm{x}}$ precursor films are stable in air. In this article, we report fabrication of epitaxial $\mathrm{Hg}-1212$ very thin film with their thickness less than $100 \mathrm{~nm}$ on (001) $\mathrm{LaAlO}_{3}$ substrates using the cationexchange process. Epitaxial Tl-1212 thin films were used as precursor films, which were annealed in $\mathrm{Hg}$ vapor to form $\mathrm{Hg}-1212$ thin film.

Manuscript received September 15, 1998.

This work was supported in part by AFOSR, NSF, NSF EPSCoR, and DEPSCoR.

\section{EXPERIMENT}

T1-1212 precursor films were prepared using dc-magnetron sputtering and post-annealing method. Non-superconducting precursor films were sputtered from a pair of superconducting Tl-1212 targets onto $\mathrm{LaAlO}_{3}(001)$ singlecrystal substrates in a mixture of $\mathrm{Ar}$ and $\mathrm{O}_{2}$ gases $(\mathrm{Ar} /$ $\mathrm{O}_{2}=4 / 1$ ) at total pressure of $20 \mathrm{mTorr}$. The as-deposited film was amorphous with the metal composition of $\mathrm{Tl}: \mathrm{Ba}: \mathrm{Ca}: \mathrm{Cu}=$ $1: 2: 1: 2$. The thickness of the films is dependent on the sputtering time and is typically controlled in the range from $50-200 \mathrm{~nm}$. The as-deposited films were annealed in $1 \mathrm{~atm} \mathrm{O}_{2}$ using crucible process at temperatures ranging from 800 $850^{\circ} \mathrm{C}$.

The precursor films of Tl-1212 were then sealed in a precleaned and evacuated quartz tube with bulk pellets of $\mathrm{Ba}_{2} \mathrm{Ca}_{2} \mathrm{Cu}_{3} \mathrm{O}_{\mathrm{x}}$ and $\mathrm{HgBa}_{2} \mathrm{Ca}_{2} \mathrm{Cu}_{3} \mathrm{O}_{\mathrm{x}}$ and annealed in $\mathrm{Hg}$ vapor at high temperature, typically $760-780^{\circ} \mathrm{C}$ for $3-4$ hours to form $\mathrm{Hg}-1212$ films. The mass ratio between the two bulk pellets was adopted to be 1:2.5 3 in order to maintain proper $\mathrm{Hg}$ vapor pressure. After the sintering, the films were further annealed at $300^{\circ} \mathrm{C}$ in a flowing $\mathrm{O}_{2}$ atmosphere for 1 hour to optimize the oxygen content of the films.

Material phase(s) and orientation were determined using $\mathrm{x}$ ray diffraction (XRD) $\theta-2 \theta$ scans from $\mathrm{Cu} \mathrm{K} \alpha$ radiation. Scanning electron microscopy (SEM) was used to determine film surface morphology. $\mathrm{T}_{\mathrm{c}} \mathrm{s}$ of the Tl-1212 films and $\mathrm{Hg}$ 1212 films was determined by both electrical transport fourprobe method and magnetic measurement. The critical current density $\left(\mathrm{J}_{\mathrm{c}}\right)$ can be estimated from magnetic measurements with the magnetic field applied perpendicular to the plane of the film.

\section{RESULTS}

The precursor thin films of Tl-1212 films are superconducting with $\mathrm{T}_{\mathrm{c}}$ about $80 \mathrm{~K}$. The surface morphology of the films observed by SEM is typically smooth and uniform. Fig. 1(a) shows the XRD spectrum of a $80 \mathrm{~nm}$ thick Tl-1212 thin film annealed at $825^{\circ} \mathrm{C}$. As indicated in Fig. 1(a), the sample is dominated by $\mathrm{T} 1-1212$ phase with a negligible portion of impurity phases. The Tl-1212 phase grows with c-axis orientation perpendicular to the substrate surface as indicated by the $(001)$ peaks. The XRD $\theta-2 \theta$ pattern of the film after $\mathrm{Hg}$-vapor annealing process is presented in Figure 1(b), which shows a nearly unchanged spectrum as expected from the same crystalline the Tl-1212 and $\mathrm{Hg}-1212$ phases have. The transformation from Tl-1212 


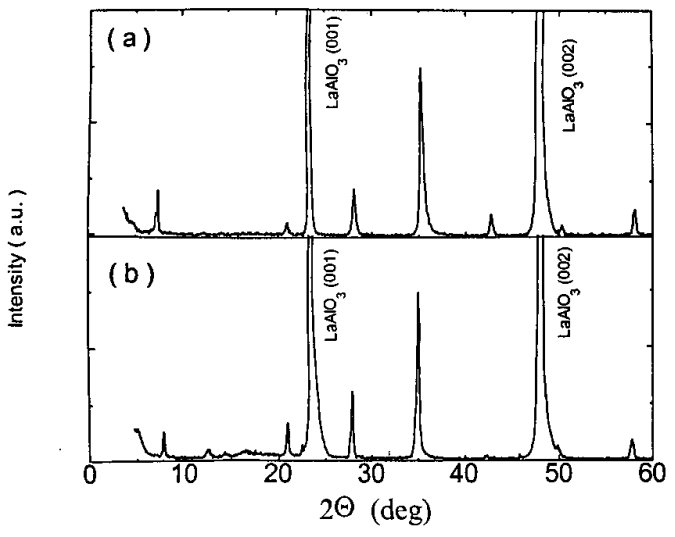

Fig. 1. The x-ray diffraction patterns for (a) the Tl-1212 film $80 \mathrm{~nm}$ thick annealed at $825^{\circ} \mathrm{C}$ and (b) the $\mathrm{Hg}-1212$ film ( $80 \mathrm{~nm}$ thick) annealed at $780^{\circ} \mathrm{C}$ for $3 \mathrm{~h}$.

to $\mathrm{Hg}-1212$ is, however, confirmed by $\mathrm{T}_{c}$ measurement. Fig. 2 shows the temperature $(\mathrm{T})$ dependence of the zero-fieldcooled (ZFC) dc magnetization (M) of the same Hg-1212 film in a 5 Gauss magnetic field measured in a superconducting quantum interference device (SQUID) magnetometer. The $\mathrm{T}_{\mathrm{c}}$ of the $\mathrm{Hg}-1212 \mathrm{film}$ is as high as 118 $\mathrm{K}$, which is close to the intrinsic value of $124 \mathrm{~K}$ for $\mathrm{Hg}-1212$ phase and is comparable to the $T_{c}(120-124 \mathrm{~K})$ obtained on thick (thickness $>200 \mathrm{~nm}$ ) Hg-1212 films [8]. It has been noticed that this $T_{c}$ value is about $38 \mathrm{~K}$ higher than that of the precursor Tl-1212 film. In addition, the smooth transition near $\mathrm{T}_{c}$ indicates a nearly complete transformation from $\mathrm{Tl}$ 1212 to $\mathrm{Hg}-1212$ phase after the $\mathrm{Hg}$-vapor annealing.

To estimate the magnitude of $\mathrm{J}_{\mathrm{c}}$ from the $\mathrm{M}-\mathrm{H}$ loops measured at different temperatures for the same $\mathrm{Hg}-1212$ film, the Bean formula was used: $J_{c}=20\left(M_{+}-M_{.}\right) / R$. Here $M_{+}$

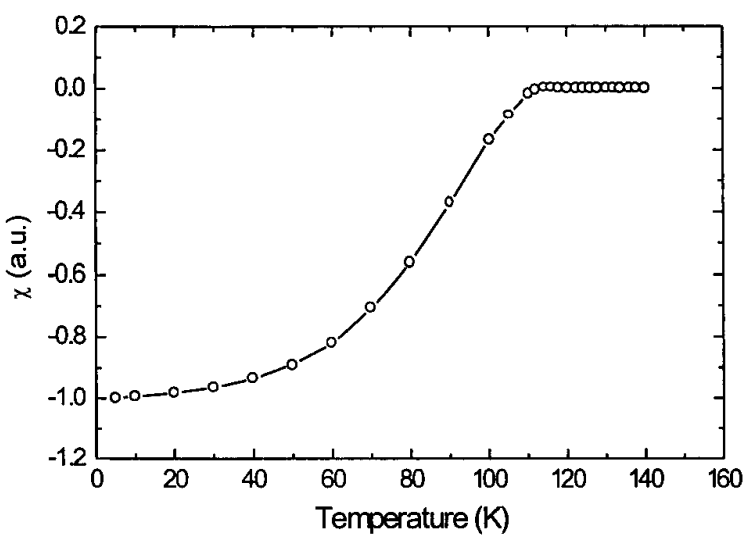

Fig. 2. Zero-field-cooled dc magnetization of ( $80 \mathrm{~nm}$-thick) $\mathrm{Hg}$ - 1212 film as a function of temperature in a $5 \mathrm{G}$ magnetic field.

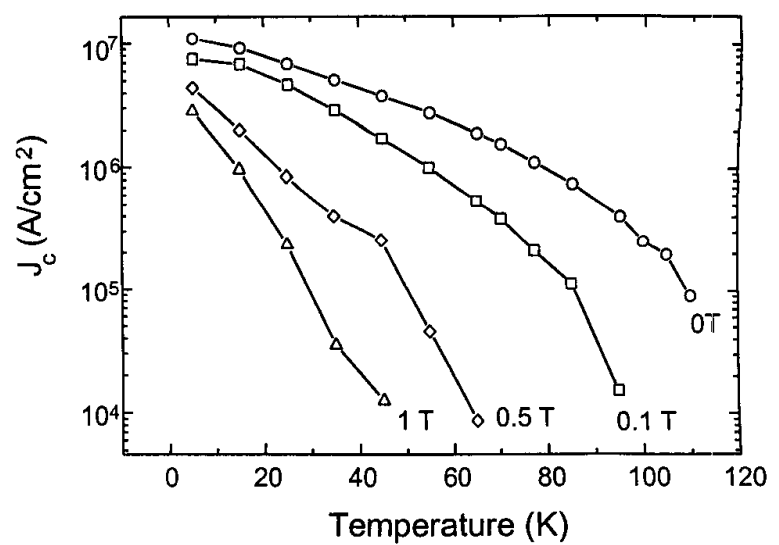

Fig. 3. $\mathrm{J}_{\mathrm{c}}$ of $\mathrm{Hg}-1212 \mathrm{film}$ (80 $\mathrm{nm}$ thick) as function of temperature and magnetic field.

and $M_{-}$are the upper and lower branches of the $\mathrm{M}-\mathrm{H}$ hysteresis loop, respectively. $R$, the circulation radius of the current, is estimated for a rectangular film sample using, $\mathrm{R}=\mathrm{b}(1-\mathrm{a} / 3 \mathrm{~b})$ where $\mathrm{a}$ and $\mathrm{b}$ represent the short and long dimensions of the sample. The calculated $\mathrm{J}_{\mathrm{c}} \mathrm{s}$ are shown in Fig. 3 as functions of the magnetic field and temperature. In calculation of the $J_{c}$ values, the total film area $(5 \mathrm{~mm}$ by 2.4 $\mathrm{mm}$ ) was used so that the calculation gives a lower bound estimates for $\mathrm{J}_{\mathrm{c}}$. At zero field, $\mathrm{J}_{\mathrm{c}}$ is $1.1 \times 10^{7} \mathrm{~A} / \mathrm{cm}^{2}$ at $5 \mathrm{~K}$ and drops to $1.14 \times 10^{6} \mathrm{~A} / \mathrm{cm}^{2}$ at $77 \mathrm{~K}$. These values are lower than the best obtained on thicker films by a factor of 2-4 [9]. At higher temperatures, the reduction of $\mathrm{J}_{\mathrm{c}}$ becomes more significant. At $100 \mathrm{~K}$ and $110 \mathrm{~K}, \mathrm{~J}_{\mathrm{c}} \mathrm{s}$ are, respectively, $2.59 \times 10^{5} \mathrm{~A} / \mathrm{cm}^{2}$ and $9.1 \times 10^{4} \mathrm{~A} / \mathrm{cm}^{2}$, which are a factor of $5-7$ lower than the best reported for a thick Hg-1212 film. Further optimization of processing conditions is necessary to improve

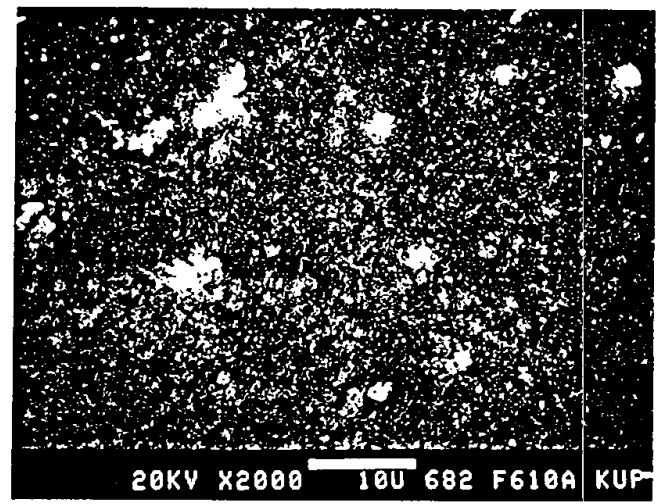

Fig. 4. SEM pictures of $80 \mathrm{~nm}$-thick $\mathrm{Hg}-1212$ film made by cation-exchange process. 
the quality of the very-thin $\mathrm{Hg}-1212$ films. On the other hand, it should be mentioned that such reduction in $T_{c}$ and $J_{c}$ is not uncommon and has been reported on other high- $T_{c}$ superconducting (HTS) very-thin films [10]. Since a protection layer could considerably decrease this reduction, it is argued that oxygen depletion near the film surface may be responsible for the degraded superconducting properties of HTS very-thin films. A comparative investigation of sample quality with or. without protection layer would provide insights in this issue.

Figure 4 shows a typical SEM image of $80 \mathrm{~nm}$-thick $\mathrm{Hg}$ 1212 films. Smooth morphology and dense crystal structure can be seen clearly on the surface. No evident grain boundary structure or cracks are visible, indicating a well-connected film at the thickness of $80 \mathrm{~nm}$.

\section{CONCLUSIONS}

Superconducting Hg-1212 films with their thickness less than $100 \mathrm{~nm}$ have been fabricated on $\mathrm{LaAlO}_{3}$ substrates by annealing epitaxial Tl-1212 precursor films in a controlled $\mathrm{Hg}$-vapor. The $\mathrm{T}_{\mathrm{c}}$ up to $118 \mathrm{~K}$ was obtained in these films. At zero applied field, $\mathrm{J}_{\mathrm{c}}$ is up to $1.1 \times 10^{7} \mathrm{~A} / \mathrm{cm}^{2}$ at $5 \mathrm{~K}$ and close to $1 \times 10^{5} \mathrm{~A} / \mathrm{cm}^{2}$ at $110 \mathrm{~K}$. These very-thin films are very promising for various microelectronic applications.

\section{REFERENCES}

[1] S.N. Putilin, E.V. Antipov, O. Chmassem, and M. Marzio, "Superconductivity at $94 \mathrm{~K}$ in $\mathrm{HgBa}_{2} \mathrm{CuO}_{4+\delta}$ ", Nature (London) 362, 226 (1993).

[2] A. Schilling, M. Cantoni, J.D. Go, and H.R. Ott, "Superconductivity above $130 \mathrm{~K}$ in the $\mathrm{Hg}-\mathrm{Ba}-\mathrm{Ca}-\mathrm{Cu}-\mathrm{O}$ system", Nature 363, 56 (1993).

[3] S.H. Yun and J.Z Wu, "Superconductivity above $130 \mathrm{~K}$ in highquality mercury-based cuprate thin films", Appl. Phys. Lett. 68, 862 (1996).

[4] S.H. Yun, J.Z. Wu, S.C. Tidrow, D.W. Eckart, "Growth of $\mathrm{HgBa}_{2} \mathrm{CaCu}_{3} \mathrm{O}_{6+\delta}$ thin films on $\mathrm{LaAlO}_{3}$ substrates using fast temperature ramping $\mathrm{Hg}$-vapor annealing", Appl. Phys. Lett. 68, 2565 (1996).

[5] F. Foong, B. Bedard, Q.L. Xu, and S.H. Liou, "C-oriented (Hg,Tl)based superconducting films with $\mathrm{T}_{\mathrm{c}}>125 \mathrm{~K}$ ", Appl. Phys. Lett. 68, 1153 (1996).

[6] C. C. Tsuei, A. Gupta, G. Trafas, and D. Mitzi, "Superconducting Mercury-Based Cuprate Films with a Zero-Resistance Transition Temperature of 124 Kelvin", Science 263, 1259 (1994).

[7] J.Z. Wu, S.H.Yun, A.Gapud, B.W.Kang, W.N. Kang, S.C. Tidrow, T.P. Monahan, X.T. Cui, and W.K. Chu, "Epitaxial Growth of $\mathrm{HgBa}_{2} \mathrm{CaCu}_{2} \mathrm{O}_{6+\delta} \delta$ thin films on $\mathrm{SrTiO}_{3}$ substrates", Physica $\mathrm{C} \mathrm{277}$, $219(1997)$.

[8] J.Z. Wu, S.L. Yan and Y.Y. Xie, "Cation Exchange: A New Scheme for Synthesis of High-Quality Epitaxial Hg-Based Superconducting Thin Films", submitted to Applied Physics Letters.

[9] S.L. Yan, Y.Y. Xie, J.Z. Wu, T. Aytug, A.A. Gapud, B.W. Kang, L. fang, M. He, S.C. Tidrow, K.W. Kirchner, J.R. Liu, W.K. Chu, "High critical current density in epitaxial $\mathrm{HgBa}_{2} \mathrm{CaCu}_{2} \mathrm{O}_{6+\delta}$ thin films", $A p p l$. Phys. Lett. 73, 2989 (1998).

[10] X.X. Xi, J. Geerk, G. Linker, Q. Li, and O. Meyer, "Preparation and superconducting properties of ultrathin $\mathrm{YBa}_{2} \mathrm{Ca}_{3} \mathrm{O}_{7 \times \mathrm{x}}$ films", $A p p l$. Phys. Lett. 54, 23 (1989). 